\title{
ORIGINAL RESEARCH ARTICLE Simulation study of pressure swing adsorption to purify helium using zeolite 13X
}

\author{
Ehsan Javadi Shokrooo $^{1,2^{*}}$, Mehdi Farniaei ${ }^{1}$, Mehdi Baghbani ${ }^{1}$ \\ ${ }^{1}$ FAPKCO Engineering Group, Elite Foundation for Southern Zone, Shiraz, Iran. E-mail: ehsan.javadi@hotmail.com; \\ mehdi.farniaei@yahoo.com; baghbani2003@yahoo.com \\ ${ }^{2}$ Faculty of Engineering, Selinus University of Sciences and Literature, 40138 Bologna BO, Italy.
}

\begin{abstract}
A two-bed pressure swing adsorption system on a commercial type of zeolite $13 \mathrm{X}$ adsorbent has been studied numerically over a wide range of operating conditions to helium separation from gaseous mixture. The model includes energy, mass and momentum balances. The coupled partial differential equations are solved using fully implicit forth order Rung-Kutta scheme in the simulation. The effects of adsorption step pressure, adsorption step time and feed flow rate on the helium purity and recovery were investigated. Results shown that as the adsorption step pressure increases the helium purity will be increased. In addition, the helium recovery increases, and the helium purity decreases when the feed flow rate increases. Finally, the simulation results indicated a very good agreement with some current literature experimental work.

Keywords: Pressure Swing Adsorption; Helium Recovery; Mathematical Modeling; Numerical Simulation; Zeolite 13X
\end{abstract}

\section{ARTICLE INFO}

Received 4 January 2020

Accepted 27 February 2020

Available online 1 March 2020

\section{COPYRIGHT}

Copyright (C) 2020 Ehsan Javadi Shokroo, et al.

EnPress Publisher LLC. This work is licensed under the Creative Commons Attribution-NonCommercial 4.0 International License (CC BY-NC 4.0).

http://creativecommons.org/licenses/ by-nc/4.0/

\section{Introduction}

Helium is a chemical element with symbol $\mathrm{He}$ and atomic number 2. It is a colorless, odorless, tasteless, non-toxic, inert and monatomic gas, which is the first in the noble gas group in the periodic table. The boiling and melting points of $\mathrm{He}$ are the lowest among all the elements.

Helium is the second lightest element and is the second most abundant element in the observable universe, being present at about $24 \%$ of the total elemental mass, which is more than 12 times the mass of all the heavier elements combined. Helium was first detected as an unknown yellow spectral line signature in sunlight during a solar eclipse in 1868 by French astronomer Jules Janssen. In 1903, large reserves of helium were found in natural gas fields in parts of the United States, which is by far the largest supplier of the gas today.

Liquid helium is used in cryogenics (its largest single use, absorbing about a quarter of production), particularly in the cooling of superconducting magnets, with the main commercial application being in MRI scanners. Helium's other industrial uses - as a pressurizing and purge gas, as a protective atmosphere for arc welding and in processes such as growing crystals to make silicon wafers, account for half of the gas produced. A well-known but minor use is as a lifting gas in balloons and airships ${ }^{[1]}$. On the Earth, it is relatively rare $5.2 \mathrm{ppm}$ by volume in the atmosphere.

Pressure swing adsorption (PSA) process is a wide operating unit to separate and purify the gases that operates based on capability of solids ad- 
sorption and selective separation of gases. The important operational parameter in this system is the pressure, and most industrial units operate at/or vicinity of the surrounding temperature. Today, the PSA process is completely known in a wide region of the processes, and this process was preferred in contrast to other conventional separation methods, especially for lower capacity and higher purity.

Linde Group developed the world's first air separation plant for the production of oxygen in 1902 and the first production facility was set up as early as 1903. Since then, it is one of the world's largest helium suppliers and since 1994 Linde has been the sole provider of helium and helium separators by the method of swing adsorption. Bhushan $^{[2]}$ purified helium by gas adsorption method in 2011 .The purifier was designed to purify up to $40 \%$ impurity to give 4.5 grade or $99.995 \%$ pure helium by high pressure and low temperature cryosorption process. Activated carbons have been used for a long time at low temperature for cryogenic applications. The pore geometry and size can be used to optimize the carbon structure for a specific application. In 1978, Stoll et al. ${ }^{[3]}$ commissioned a fully automatic large capacity helium purifier which included operation, regeneration, recooling and re-pressurization time. In 2007, E. Van Cleve et al. ${ }^{[4]}$ developed a cryogenic pulsed laser deposition (PLD) system to deposit lithium films onto a quartz crystal microbalance (QCM) and adsorption isotherms of $4 \mathrm{He}$ on lithium were measured. PLD system was used to form lithium substrates and the first helium adsorption measurements on this surface were reported. In 2008, Nisith $\mathrm{Kr}$. Das ${ }^{[1]}$ employed a technique known as pressure swing adsorption to concentrate a lean amount of helium present in natural gas through selective physical elimination of $\mathrm{N}_{2}, \mathrm{CO}_{2}, \mathrm{CH}_{4}$ and heavier hydrocarbons in a stepwise cycle sequence at present time intervals. In 2011, D. Martins et al. ${ }^{[5]}$ reported on the low temperature adsorption properties of $\mathrm{He}, \mathrm{H}_{2}$, and $\mathrm{N}_{2}$, using three activated carbons with different pore size distributions. In 2012, R. Majidia et al. ${ }^{[6]}$ used the molecular dynamics simulation to study the helium adsorption on the CNCs with a declination angle of $240^{\circ}$ and $300^{\circ}$. The results indicated that the adsorption capacity of the CNCs became considerable by decreasing the declination angle. In the same year, Nisith Kr. Das et $a l^{[7]}$ developed a helium purification system using a three-bed seven-step pressure swing adsorption (PSA). It removed impurities like $\mathrm{N}_{2}$ and $\mathrm{O}_{2}$ from a ternary mixture leaving out high-purity helium from the gas mixture. The PSA system operated successfully resulting in high-purity helium (>99.9\%) with a yield of around $89 \%$. The adsorption capability of carbon dioxide on 5A molecular sieve (5AMS) was investigated in a fixed-bed apparatus with two-road gas mixing system by dynamic column breakthrough method with helium as the carrier gas for helium purification system of high-temperature gas-cooled reactor (HTGR) in 2013 by Chang Hua et al. ${ }^{[8]}$

In 2014, Bartolomei et al. ${ }^{[9]}$ performed quantum dynamical simulations on reliable new force fields in order to assess the graphdiyne capability for helium chemical and isotopic separation.

In this work, a semi-industrial PSA unit for helium recovery from gaseous mixture was simulated. In this process, the effect of some operating variables such as adsorption time, feed flow rate, and adsorption pressure on process performance was investigated. The simulated PSA process is a six-step process with the following sequence (Figure 1): (I) co-current feed pressurization (PR); (II) high-pressure adsorption (AD) step; (III) counter-current depressurizing pressure equalization (ED) step; (IV) counter-current blow down (BD) step; (V) counter-current purge with a light product (PG) step; (VI) co-current pressurizing pressure equalization (EP) step. The sequence time table of the PSA process was also depicted in Table 1. 
Table 1. Step sequence of the PSA process

\begin{tabular}{lllllll}
\hline No. & 1 & 2 & 3 & 4 & 5 & 6 \\
Time (sec.) & 25 & 60 & 10 & 25 & 60 & 10 \\
Column 1 & PR & AD & ED & BD & PG & EP \\
Column 2 & BD & PG & EP & PR & AD & ED \\
\hline SV1 & on & on & off & on & on & off \\
SV2 & off & off & on & off & off & off \\
SV3 & off & off & off & off & off & on \\
SV4 & on & on & off & on & on & off \\
SV5 & on & on & off & on & on & off \\
SV6 & on & on & off & on & on & off \\
SV7 & off & off & off & off & on & off \\
SV8 & off & on & off & off & off & off \\
SV9 & off & off & off & off & on & off \\
SV10 & off & on & off & off & off & off \\
SV11 & off & on & off & off & on & off \\
SV12 & off & on & off & off & off & off \\
SV13 & off & off & off & on & on & off \\
\hline
\end{tabular}

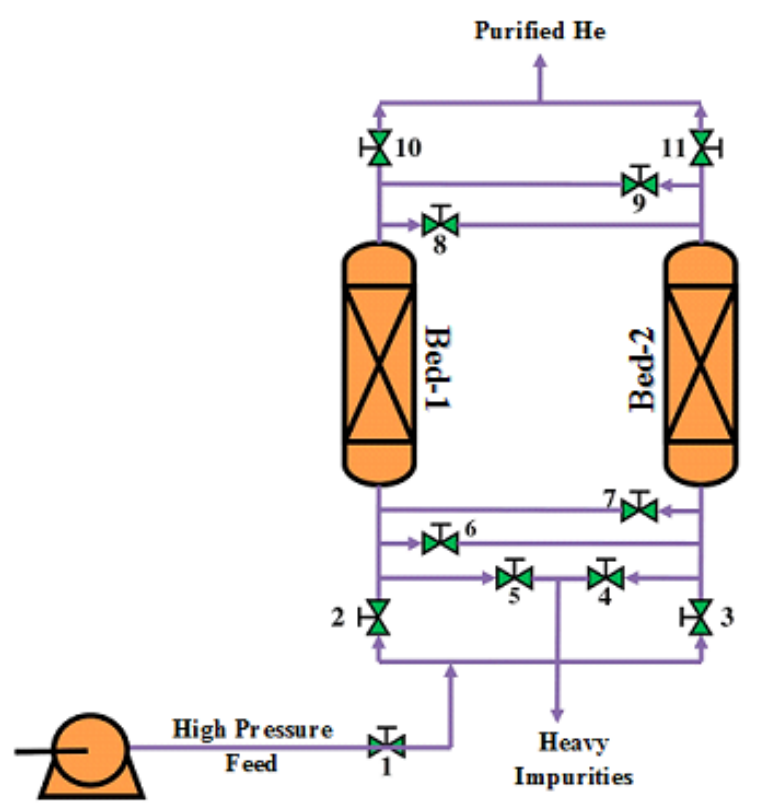

Figure 1. Schematic diagram of He-PSA.

\section{Mathematical model}

In order to develop a mathematical model for an adsorption bed, the following assumptions were made:

1- Gas behaves as an ideal gas;

2- The flow pattern is axially assumed as plugflow model;

3- Equilibrium equations for air are expressed as multi-component Langmuir-Freundlich isotherm;

4- Rate of mass transfer is presented by linear driving force (LDF) relations;

5- Bed is clean at initial state and there is no gas flow in it;

According to these assumptions, dynamic be- havior of system in terms of mass, energy and momentum balances can be expressed as follows:

Dimensionless partial mass balance for gas phase in the adsorption bed is ${ }^{[9-12]}$ :

$$
\begin{gathered}
-\left(\frac{1}{P_{e}^{m}}\right) \cdot \frac{\partial^{2} y_{i}}{\partial \hat{z}^{2}}+y_{i} \cdot \frac{\partial \widehat{u}}{\partial \hat{z}}+\hat{u} \cdot\left(\frac{\partial y_{i}}{\partial \hat{z}}+y_{i} \cdot\left(\frac{1}{\hat{P}} \cdot \frac{\partial \hat{P}}{\partial \hat{z}}-\frac{1}{\hat{T}} \cdot \frac{\partial \hat{T}}{\partial \hat{z}}\right)\right)+\frac{\partial y_{i}}{\partial \tau}+ \\
y_{i} \cdot\left(\frac{1}{\hat{P}} \cdot \frac{\partial \hat{P}}{\partial \tau}-\frac{1}{\hat{T}} \cdot \frac{\partial \hat{T}}{\partial \tau}\right)+\left(\frac{\rho_{p} \cdot R \cdot T_{0} \cdot \hat{T}}{P_{0} \cdot \hat{P}}\right) \cdot\left(\frac{1-\varepsilon}{\varepsilon}\right) \cdot\left(q_{m, i} \cdot \frac{\partial \hat{q}_{i}}{\partial \tau}+\hat{q}_{i} \cdot \frac{\partial q_{m, i}}{\partial \tau}\right)=0
\end{gathered}
$$

Dimensionless equilibrium loading of ith component for solid phase in the adsorption bed is:

$$
\frac{\partial q_{m, i}}{\partial \tau}=\frac{\partial q_{m, i}}{\partial \hat{T}} \times \frac{\partial \hat{T}}{\partial \tau}=k_{2, i} T_{0} \times \frac{\partial \hat{T}}{\partial \tau}
$$

Dimensionless loading of ith component for solid phase in the adsorption bed is (LDF relation):

$$
\frac{\partial \hat{q}_{i}}{\partial \tau}=\alpha_{i} \cdot\left(\frac{\beta_{i} \cdot y_{i}^{n i}}{1+\sum_{j-1}^{N} \beta_{j} \cdot y_{j}{ }^{n j}}-\hat{q}_{i}\right)-\left(\frac{\widehat{q}_{i}}{q_{m, i}} \cdot \frac{\partial q_{m, i}}{\partial \tau}\right)
$$

According to equation (3), the LDF relation depends on various parameters such as: equilibrium parameter for the Langmuir model, mole fraction of species $i$ in the gas phase, average amount adsorbed and equilibrium parameter for the Langmuir model.

The equilibrium of triple Langmuir-Freundlich isotherm is as follows:

$$
\hat{q}_{i}^{*}=\frac{\beta_{i} y_{i}^{n i}}{1+\sum_{j-1}^{N} \beta_{j} \cdot y_{j}^{n j}}
$$

where $\beta, n$ and $q_{m}$ are as follows:

$$
\begin{aligned}
& q_{m, i}=k_{1}+k_{2} T_{0} \widehat{T} \\
& \beta_{i}=k_{3} \exp \left(\frac{k_{4}}{T_{0} \widehat{T}}\right) \\
& n=k_{5}+\frac{k_{6}}{T_{0} \hat{T}}
\end{aligned}
$$

Overall dimensionless mass balance for gas phase in the adsorption bed is ${ }^{[13-16]}$ :

$$
\begin{aligned}
& \left(\frac{1}{\hat{P}}\right) \cdot \frac{\partial \hat{P}}{\partial_{\tau}}+\frac{\partial \hat{u}}{\partial \hat{z}}+\frac{\hat{u}}{\hat{P}} \cdot \frac{\partial \hat{P}}{\partial \hat{Z}}-\left(\frac{1}{\hat{T}}\right) \cdot\left(\frac{\partial \hat{T}}{\partial_{\tau}}+\hat{u} \frac{\partial \hat{T}}{\partial \hat{z}}\right)+ \\
& \left(\frac{P_{p} R T_{0} \hat{T}}{P_{0} \hat{P}}\right) \cdot\left(\frac{1-\varepsilon}{\varepsilon}\right) \cdot \sum_{i-1}^{3}\left(q_{m, i} \cdot \frac{\partial \widehat{q}_{l}}{\partial_{\tau}}+\hat{q}_{i} \cdot \frac{\partial q_{m, i}}{\partial_{\tau}}\right)=0
\end{aligned}
$$

Dimensionless energy balance for gas phase in the adsorption bed is ${ }^{[17-21]}$ : 
$-\left(\frac{1}{P_{e}^{h}}\right) \cdot \frac{\partial^{2} \hat{T}}{\partial \hat{z}^{2}}+\varepsilon \cdot\left(\hat{\mathrm{u}} \frac{\partial T}{\partial \hat{\mathrm{z}}}+\widehat{T} \frac{\partial \hat{u}}{\partial \hat{z}}\right)+\left(\varepsilon_{t}+\frac{\rho_{B} \cdot c_{p, s}}{\rho_{g} \cdot c_{p, g}}\right) \cdot \frac{\partial \widehat{T}}{\partial \tau}-\left(\frac{\rho_{B}}{T_{0} \cdot \rho_{g} \cdot c_{p, g}}\right) \cdot$
$\sum_{i=1}^{3}\left[\left(q_{m, i} \cdot \frac{\partial \hat{q}_{i}}{\partial \tau}+\hat{q}_{i} \cdot \frac{\partial q_{m, i}}{\partial \tau}\right) \cdot\left(-\Delta \bar{H}_{i}\right)\right]+\left(\frac{2 h_{i} \cdot L}{R_{B, i} \cdot U_{0} \cdot \rho_{g} \cdot c_{p, g}}\right) \cdot\left(\widehat{\mathrm{T}}-\widehat{T}_{w}\right)=0$

Dimensionless energy balance for the wall of adsorption bed is:

$\frac{\partial \widehat{\mathrm{T}}_{w}}{\partial \tau}=\left[\frac{2 \pi \cdot R_{B, i} \cdot h_{i} \cdot L}{\rho_{w} \cdot c_{p, w} \cdot A_{w} \cdot U_{0}}\right] \cdot\left(\widehat{\mathrm{T}}-\widehat{\mathrm{T}}_{w}\right)-\left[\frac{2 \pi \cdot R_{B, o} \cdot h_{o} \cdot L}{\rho_{w} \cdot c_{p, w} \cdot A_{w} \cdot U_{0}}\right] \cdot\left(\widehat{\mathrm{T}}_{w}-\frac{T_{a t m}}{T_{0}}\right)$

Cross-sectional area of adsorption bed wall is:

$$
A_{w}=\pi \cdot\left(R_{B, o}^{2}-R_{B, i}^{2}\right)
$$

Ergun equation is utilized in order to investigate the pressure drop across the tion bed ${ }^{[22,23]}$.

$$
\begin{aligned}
& -\frac{d \hat{P}}{d \hat{z}}=\left[a \cdot \mu \cdot U_{0} \cdot \hat{u}+b \cdot \rho \cdot U_{0}^{2} \cdot \hat{u} \cdot|\hat{u}|\right] \cdot\left(\frac{L}{P_{0}}\right) \\
& a=\frac{150}{4 R_{p}^{2}} \cdot \frac{(1-\varepsilon)^{2}}{\varepsilon^{2}} ; b=1.75 \frac{(1-\varepsilon)}{2 R_{p} \varepsilon}
\end{aligned}
$$

Physical properties of adsorbents and characteristics of adsorption bed are depicted in Tables 2 and $\mathbf{3}$, respectively.

Table 2. Physical properties of bed and adsorbent ${ }^{[24]}$

\begin{tabular}{ll}
\hline Characteristic & Zeolite 13X \\
\hline Type & Sphere \\
Average pellet size, $\mathrm{R}_{\mathrm{P}}(\mathrm{cm})$ & 0.07 \\
Pellet density, $\rho_{\mathrm{p}}\left(\mathrm{g} / \mathrm{cm}^{3}\right)$ & 1.17 \\
Heat capacity, $\mathrm{C}_{\mathrm{ps}}(\mathrm{cal} / \mathrm{g} . \mathrm{K})$ & 0.32 \\
Bed porosity, $\varepsilon$ & 0.391 \\
Bed density, $\rho_{\mathrm{B}}\left(\mathrm{g} / \mathrm{cm}^{3}\right)$ & 0.713 \\
\hline
\end{tabular}

Table 3. Adsorption bed properties ${ }^{[25]}$

\begin{tabular}{ll}
\hline Characteristic & Zeolite 13X \\
\hline Length, $\mathrm{L}(\mathrm{cm})$ & 76 \\
Inside radius, $\mathrm{R}_{\mathrm{Bi}}(\mathrm{cm})$ & 2.138 \\
Outside radius, $\mathrm{R}_{\mathrm{Bo}}(\mathrm{cm})$ & 2.415 \\
Heat capacity of the column, $\mathrm{C}_{\mathrm{pw}}(\mathrm{cal} / \mathrm{g} . \mathrm{K})$ & 0.12 \\
Density of column, $\rho_{\mathrm{w}}\left(\mathrm{g} / \mathrm{cm}^{3}\right)$ & 7.83 \\
Internal heat-transfer coefficient, $\mathrm{h}_{\mathrm{i}}$ & $9.2 \times 10^{-4}$ \\
$\left(\mathrm{cal} / \mathrm{cm}^{2} . \mathrm{K} . \mathrm{s}\right)$ & \\
External heat-transfer coefficient, $\mathrm{h}_{\mathrm{o}}$ & $3.4 \times 10^{-4}$ \\
$\left(\mathrm{cal} / \mathrm{cm}^{2} . \mathrm{K} . \mathrm{s}\right)$ & $6.2 \times 10^{-5}$ \\
Axial thermal conductivity, $\mathrm{K}_{\mathrm{L}}(\mathrm{cal} / \mathrm{cm} . \mathrm{s} . \mathrm{K})$ & $1 \times 10^{-5}$ \\
Axial dispersion coefficient, $\mathrm{D}_{\mathrm{L}}\left(\mathrm{cm}^{2} / \mathrm{s}\right)$ & \\
\hline
\end{tabular}

\section{Results and discussion}

The fourth order Runge-Kutta Gill scheme was used to solve a mathematical model considered as coupled partial differential equations. The experimental data obtained from literatures has been simulated in order to validate the simulation results in this study ${ }^{[10,22,26]}$. An experimental and simulation study of a PSA unit which is running a traditional Skarstrom cycle and a Skarstrom cycle with co-current equalization owing to separate oxygen from air using a 5A zeolite has been proposed by Mendes et $a l .{ }^{[11]}$ in 2001. Moreover, a small-scale two-bed six-step PSA process using zeolite 13X was performed by Jee et al. ${ }^{[27-30]}$ in order to provide oxygen-enriched air. They showed that there is a strong effect of feed flow rate on $\mathrm{O}_{2}$ purity. The effects of adsorption and desorption on zeolite 5A and CMS beds were investigated in a mixture of $\mathrm{N}_{2} / \mathrm{O}_{2} / \mathrm{Ar}$ by Jee et al. ${ }^{[28]}$ in 2004 . A non-isothermal mathematical model was applied in order to simulate the adsorption dynamics in their studies ${ }^{[26]}$.

Figures 2(a) and (b) indicate the effect of product flow rate and P/F on the purity and recovery of oxygen during PSA process, respectively. The impact of temperature variations in gas phase during adsorption as a function of time is illustrated in Figure 2(c). It is obviously seen that there is a relatively high accuracy in the simulation of experimental data ${ }^{[27]}$.

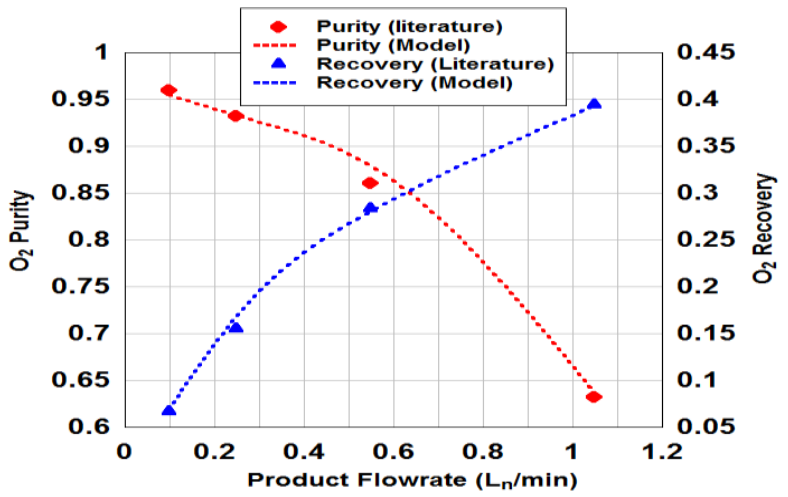

Figure 2a. Numerical simulation of experimental data in this work $^{[23]}$.

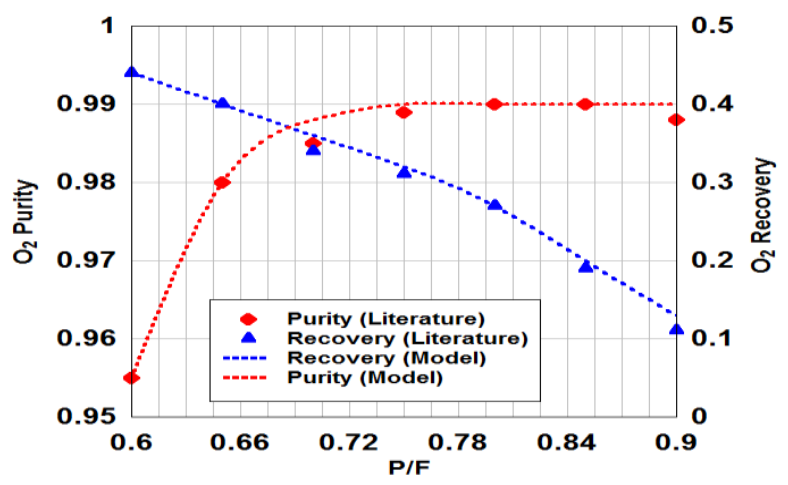

Figure 2b. Numerical simulation of experimental data in this work $^{[10]}$. 


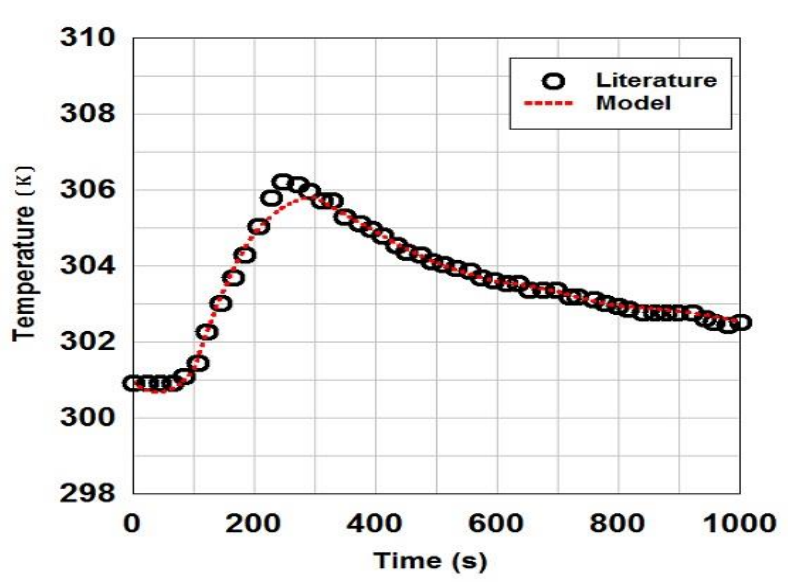

Figure 2c. Numerical simulation of experimental data in this $\operatorname{work}^{[26]}$.

Figure 3 shows the effects of feed flow rate on helium purity and recovery at adsorption pressure of 8.5 bar and adsorption time of $20 \mathrm{sec}$. In this figure it can be seen that the increase in feed leads to a decrease in the helium purity while helium recovery has increased. The feed flow will rise at a constant purge flow in these simulations, which the P/F decreases and subsequently helium purity decreases. At a certain purge flow rate when the feed amount increases therefore, the $\mathrm{P} / \mathrm{F}$ ratio is reduced and finally, the product purity decreases. In the other word, dead space of the bed in the adsorption step will rise with the feed flow rate. Thus, purging the bed in the purge step requires more purgative flow. In these simulations, a constant purge flow rate causes a reduction in the helium purity with the feed flow rate.

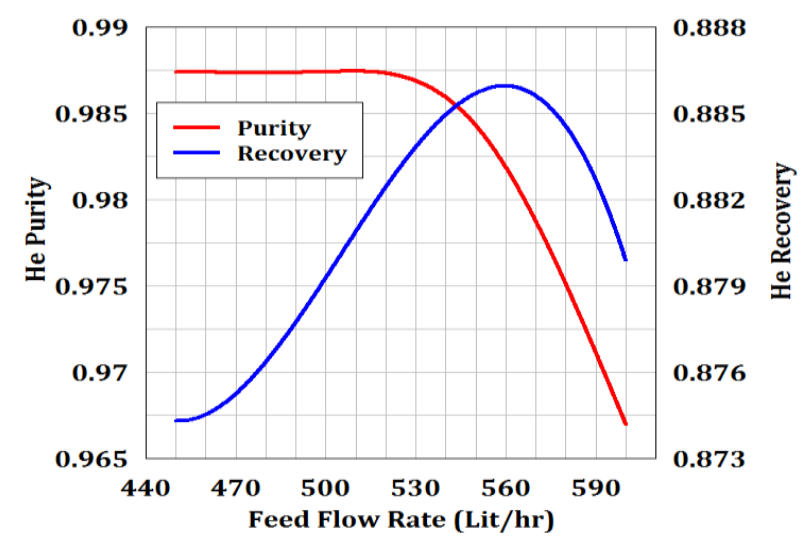

Figure 3. Helium purity and recovery variations versus feed flow rate.

The variations of helium purity and recovery in terms of adsorption step time at pressure of 5.5 bar and P/F of 0.1 are shown in Figure 4. It is clear from this figure that increase of adsorption time leads to reduction in helium purity. It is due to the well-known breakthrough time of the adsorption beds. Thus, the adsorption time should be near to the breakthrough time in order to achieve the maximum process performance in terms of adsorption time. In fact, the adsorption time is a required time for occurring breakthrough time. After this time, the product purity is decreased while the entire capacity of the bed has not utilized before this time. Therefore, the adsorption time must be close to the breakthrough time in order that the best process performance in terms of adsorption time is achieved $^{[10]}$. With referring to this figure, the best time for adsorption is $25 \mathrm{sec}$. Furthermore, it should be noted that, the helium recovery is in reverse order with its purity at all points. It was evident that the recovery reduces as the gas volume of the feed increases through increasing the adsorption time. Figure 5 shows the effect of adsorption step pressure on the process performance. It is apparent from this figure that the higher adsorption pressure improves the PSA unit performance. As the adsorption pressure increases, the amount of adsorbed heavy species on the adsorbent will be increased and therefore, the helium purity increases. For the favorable isotherm systems, if the pressure is increased, the highly adsorbed species are more adsorbed and the product purity will be increased. This result was seen in the literature $^{[10-12,15,16,21,26,31,32]}$. Helium concentration profile curves are depicted in Figure 6. It is obvious from this figure that the helium purity has a minimum and maximum quantity in the blow down step because of depressurized bed and the pressurization step as result of the cleansed bed, respectively.

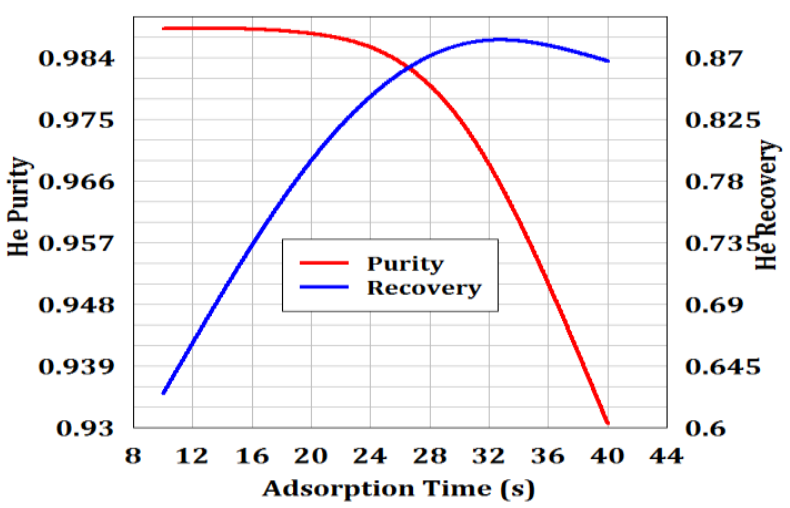

Figure 4. Variations of helium purity and recovery in terms of adsorption step time. 


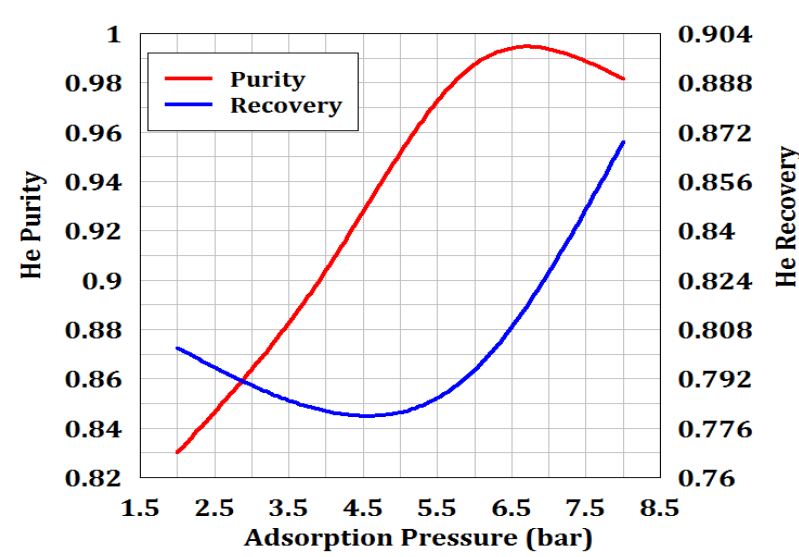

Figure 5. Variations of helium purity and recovery in terms of adsorption step pressure.

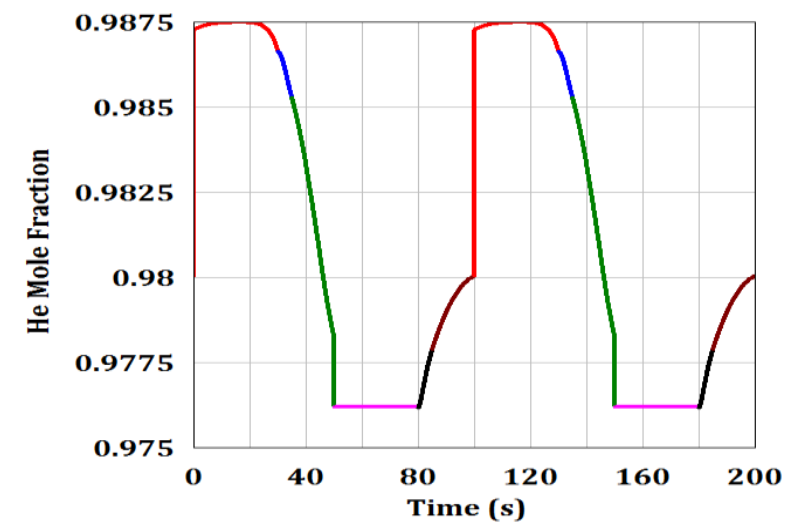

Figure 6. Helium concentration profile along a whole cycle at the top of the bed.

\section{Conclusions}

Helium separation from gaseous mixture in a two-bed pressure swing adsorption setup on a commercial type of zeolite $13 \mathrm{X}$ adsorbent has been studied numerically over a wide range of operating conditions. The influences of adsorption step pressure, adsorption step time and feed flow rate on the process performance were investigated. Results showed that as the adsorption step pressure increases, the helium purity will be increased. The time of the adsorption step is clearly defined through the physical properties of the bed such as length, diameter, adsorbent type as well as the feed flow rate. Furthermore, the helium recovery increases, and the helium purity decreases when the feed flow rate increases. Finally, a considerable agreement was found between the experimental data and the simulation results for various operating variables.

\section{Conflict of interest}

The authors declare that they have no conflict of interest.

\section{References}

1. Das NKr, Chaudhuri H, Bhandari RK, et al. Purification of helium from natural gas by pressure swing adsorption. Current Science 2008; 95(12): 1684-1687.

2. Bhushan J. Helium purification by gas adsorption method master of technology [MSc thesis].

Rourkela: National Institute of Technology; 2011.

3. Stoll AP, Taylor LG, Steel AJ. Helium purifiers. Proceedings of the seventh international cryogenic engineering conference (ICEC7); 1978. p. 642-647.

4. Van Cleve E, Taborek P, Rutledge JE. Helium adsorption on lithium substrates. Journal of Low Temperature Physics 2008; 150: 1-11.

5. Martins D, Catarino I, Lopes D, et al. Low temperature adsorption versus pore size in activated carbons. International Cryocooler Conference, Inc., Boulder, CO. 2011.

6. Majidia R, Ghanbarzadeh S, Jodaee asl N. Molecular dynamics simulation of helium adsorption on carbon nanocones with disclination angles of $240^{\circ}$ and $300^{\circ}$. The $4^{\text {th }}$ international conference on nanostructures (ICNS4); 2012.

7. Das NKr, Kumar P, Mallik C, et al. Development of a helium purification system using pressure swing adsorption. Current Science 2012; 103(6): 631-634.

8. Chang Hua, Wu Z, Yao M, et al. Experimental investigation and modeling of adsorption of carbon dioxide on $5 \mathrm{~A}$ molecular sieve for helium purification of high-temperature gas-cooled reactor. Energy Procedia 2013; 39(9): 208-226.

9. Bartolomei M, Carmona-Novillo E, Hernández MI, et al. Graphdiyne pores: "Ad Hoc" openings for helium separation applications The Journal of Physical Chemistry 2014; 118(51): 29966-29972.

10. Ruthven DM. Principle of adsorption and adsorption processes. New York: John Wiley \& Sons, Inc. 1984.

11. Mendes AMM, Costa AVC, Rodrigues AE. Analysis of nonisobaric steps in nonlinear bicomponent pressure swing adsorption systems. Application to Air Separation Industrial \& Engineering Chemistry Research 2000; 39(1): 138-145.

12. Mendes AMM, Costa AVC, Rodrigues AE. Oxygen separation from Air by PSA: modeling and experimental results part I: Isothermal operation. Separation and Purification Technology 2001; 24(1): 173-188.

13. Milton RM (inventors). UNION CARBIDE CORP (assignee). Molecular sieve adsorbents. U.S. patent. 2,882,244. 1959 Apr.

14. Yang RT. Adsorbents: Fundamentals and applications. New Jersey: John Wiley \& Sons, Inc.; 2003.

15. Ritter JA, Liu Y. Tapered pressure swing adsorption columns for simultaneous air purification and solvent vapor recovery. Industrial \& Engineering Chemistry Research1998; 37(7): 2783-2791.

16. Rege SU, Yang R, Qian K, et al. 
Air-prepurifcation by pressure swing adsorption using single/layered beds. Chemical Engineering Science 2001; 56(8): 2745-2759.

17. Mivechian A, Pakizeh M. Hydrogen recovery from Tehran refinery off-gas using pressure swing adsorption, gas absorption and membrane separation technologies: Simulation and economic evaluation. Korean Journal of Chemical Engineering 2013; 30(4): 937-948.

18. Jang SC, Yang S, Oh SG, et al. Adsorption dynamics and effects of carbon to zeolite ratio of ered beds for multi component gas adsorption. Korean Journal of Chemical Engineering 2011; 28(2): 583-590.

19. Kim YH, Lee DG, Moon DK, et al. Effect of bed void volume on pressure vacuum swing adsorption for air separation. Korean Journal of Chemical Engineering 2014; 31(1): 132-141.

20. Ruthven DM, Farooq S, Knaebel KS. Pressure swing adsorption. New York: VCH Publications Inc.; 1994.

21. Teague KG, Edgar TF. Predictive dynamic model of a small pressure swing adsorption. Industrial \& Engineering Chemistry Research 1999; 38(10): 3761-3775.

22. Zaman M, Lee JH. Carbon capture from stationary power generation sources: A review of the current status of the technologies. Korean Journal of Chemical Engineering 2013; 30(8): 1497-1526.

23. Hoshyargar V, Fadaei F, Ashrafizadeh SN. Mass transfer simulation of nanofiltration membranes for electrolyte solutions through generalized Maxwell-Stefan approach. Korean Journal of Chemical Engineering 2015; 32: 1388-1404.

24. Grande CA. Advances in pressure swing adsorption for gas separation. International Scholarly Research Notices Chemical Engineering 2012; 1-13.

25. Jee JG, Lee JS, Lee CH. Air separation by small-scale two-bed medical $\mathrm{O}_{2}$ pressure swing adsorption. Industrial \& Engineering Chemistry Research 2001; 40(16): 3647-3658.

26. Chou CT, Chen L. Simulation of a four-bed pressure swing adsorption process for oxygen enrichment. Industrial \& Engineering Chemistry Research 1994; 33(5): 1250-1258.

27. Jee JG, Park HJ, Haam SJ, et al. Effects of nonisobaric steps and isobaric steps on $\mathrm{O}_{2}$ pressure swing adsorption for an aerator. Industrial \& Engineering Chemistry Research 2002; 41(17): 4383-4392.

28. Jee JG, Lee SJ, Lee CH. Comparison of the adsorption dynamics of air on Zeolite 5A and carbon molecular sieve beds. Korean Journal of Chemical Engineering 2004; 21(6): 1183-192.

29. Jee JG, Park MK, Yoo HK, et al. Adsorption and desorption characteristics of air on zeolite 5a, 10x, and 13x fixed beds. Separation Science and Technology 2002; 37(15): 3465-3490.

30. Lee SJ, Jung JH, Moon JH, et al. Parametric study of the three-bed pressure-vacuum swing adsorption process for high purity $\mathrm{O}_{2}$ generation from ambient air. Industrial \& Engineering Chemistry Research 2007; 46(11): 3720-3728.

31. Lin L. Numerical simulation of pressure swing adsorption process [MSc thesis]. Xi'an, China: Xidian University; 1990.

32. Wilson SJ, Beh CCK, Webley PA, et al. The effects of a readily adsorbed trace component (water) in a bulk separation PSA process: The case of oxygen VSA. Industrial \& Engineering Chemistry Research 2001; 40(12): 2702-2713. 